\title{
Trauma hepático: prevalência e características epidemiológicas de vítimas encaminhadas ao Instituto Médico Legal de Palmas, Tocantins
}

\author{
Liver trauma: prevalence and epidemiological characteristics of victims referred \\ to the Forensic Department of Palmas, Tocantins
}

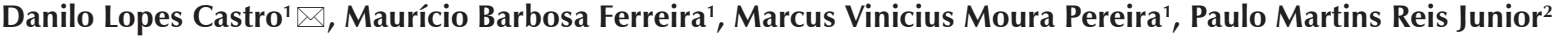 \\ ${ }^{1}$ Graduandos do sexto ano do Curso de Medicina da Universidade Federal do Tocantins (UFT), Palmas, TO. \\ 2 Professor Auxiliar do Curso de Medicina da UFT. Diretor do Instituto Médico Legal, Palmas, TO.
}

\section{RESUMO}

Objetivos: Analisar a prevalência e as características epidemiológicas relacionadas ao trauma hepático nas vítimas encaminhadas ao Instituto Médico Legal de Palmas, Tocantins.

Métodos: Um estudo transversal retrospectivo foi realizado no Instituto Médico Legal (IML) de Palmas, Tocantins, incluindo todas as vítimas encaminhadas para esse serviço, no período de janeiro de 2006 a dezembro de 2010, consistindo em óbitos por causas violentas e que possuíssem lesões traumáticas de tecido hepático. A coleta de dados foi realizada mediante análise dos registros do IML. Foram anotados sexo, idade, mecanismo do trauma (quando acidentes de trânsito, o tipo de veículo envolvido), tipos de trauma associados ao trauma abdominal, presença de lesões abdominais associadas ao trauma hepático, índice de gravidade de lesões conforme a Associação Americana de Cirurgia do Trauma e número de óbitos causados diretamente pelo trauma hepático. A análise estatística incluiu as frequências absolutas e relativas das variáveis.

Resultados: Das 1.796 vitimas fatais, 241 (13,4\%) delas apresentavam trauma hepático, entre os quais 85,5\% eram do sexo masculino e 42,9\% tinham entre 21-30 anos. A principal causa envolvida foram os acidentes de trânsito, em $143(59,3 \%)$ dos casos, envolvendo mais comumente as motos, 53 (37\%). O tipo de trauma mais encontrado foi o contuso, $179(74,3 \%)$. O lobo hepático direito foi o mais acometido, 98 (40,7\%). As vítimas eram politraumatizadas em 147 (61\%) dos casos. A lesão esplênica foi a mais associada ao trauma hepático, observada em 65 $(27 \%)$ dos avaliados. O grau de lesão hepática mais prevalente foi o grau II, presente em $102(42,4 \%)$ casos. O número de óbitos que tiveram como causa direta o trauma hepático correspondeu a $21(8,7 \%)$ de todos os casos.

Conclusões: Entre os casos de trauma hepático observados em óbitos por causas violentas, registrados no IML de Palmas, o mais comum foi do tipo contuso, decorrente principalmente de acidentes de trânsito (especialmente com motocicletas). As vítimas mais frequentes com trauma hepático foram homens jovens na faixa etária laboral.

DESCRITORES: fígado; ferimentos e lesões; traumatologia; epidemiologia; acidentes de trânsito.

\section{ABSTRACT}

Aims: To assess the prevalence and epidemiological characteristics of hepatic trauma in victims referred to the Forensic Department of Palmas, State of Tocantins, Brazil.

Methods: A retrospective cross-sectional study was performed with all the victims referred to the Forensic Department of Palmas from January 2006 to December 2010, to investigate violent deaths with severe damage to the liver tissue. The data were collected from the Forensic Department records. The following data were evaluated: sex, age, mechanism of injury (in case of motor vehicle accidents, the type of vehicle involved), types of trauma associated with abdominal trauma, presence of abdominal lesions associated with hepatic injury, the American Association for the Surgery of Trauma Organ Injury Scale, and number of deaths caused directly by hepatic trauma. The statistical analysis included absolute and relative frequencies of the variables.

Results: A total of 241 (13.4\%) out of 1,796 victims had liver trauma, among whom $85.5 \%$ were male and $42.9 \%$ belonged to the $21-30$ year age group. Motor vehicle accidents were the major cause of trauma in 143 cases, $(59.3 \%)$, involving mainly motorcycles (53 cases or $37 \%)$. Blunt trauma was the most frequent mechanism of injury, having occurred in 179 cases $(74.3 \%)$. The right lobe was injured in 98 cases $(40.7 \%)$. A total of 147 victims $(61 \%)$ had polytrauma. Splenic injury, present in $65(27 \%)$ of the cases, was mostly associated with liver trauma. Liver injury of grade II was detected in 102 cases (42.4\%) and was therefore the most prevalent type of damage. Twenty-one (8.7\%) deaths were directly related to liver trauma.

Conclusions: Blunt injury, caused mainly by motor vehicle accidents (especially with motorcycles), was the most common type of liver trauma observed in violent deaths, according to the records of the Forensic Department of Palmas. Young men of working age were the most frequent victims with liver trauma.

KEY WORDS: liver; wounds and injuries; traumatology; epidemiology; accidents, traffic. 


\section{INTRODUÇÃO}

O traumatismo é um relevante problema de saúde pública no mundo, sendo um dos principais motivos de mortalidade e morbidade em países desenvolvidos, acometendo principalmente indivíduos com menos de 40 anos de idade, com isso gerando grande impacto econômico e social $[1,2]$. O fígado está acometido em aproximadamente $5 \%$ das admissões por trauma em serviço de urgência, correspondendo a um dos órgãos mais afetados, principalmente devido ao seu tamanho e sítio anatômico, apresentando o trauma abdominal fechado como o principal mecanismo de lesão [2-4].

A Associação Americana de Cirurgia do Trauma propõe uma escala de classificação das lesões traumáticas conforme a gravidade da lesão: a American Association for the Surgery of Trauma-Organ Injury Scale (AAST-OIS), imprescindível para a comparação entre os vários centros de trauma e, também, entre diferentes períodos $[5,6]$. Nos traumas que envolvem o fígado, o choque hemorrágico, devido ao sangramento incontrolável dos vasos e do parênquima, é a principal causa de morte nas primeiras 36 horas após a lesão, enquanto mais tardiamente, as complicações sépticas decorrentes da formação de abscesso em áreas de necrose do parênquima hepático, assim como a ruptura de ductos biliares, representam as principais causas de complicações [7]. As taxas de mortalidade são bastante altas, variando entre 10 e $30 \%$ para trauma fechado e 5 a $10 \%$ para trauma hepático penetrante. A mortalidade em pacientes com trauma hepático situase entre 4 e $15 \%$, dependendo do tipo de lesão e da afecção simultânea de outros órgãos [4].

A pesquisa em relação ao trauma hepático tem uma grande importância, uma vez que permite o entendimento dos mecanismos de lesão hepática, o conhecimento da taxa de morbimortalidade e a avaliação dos riscos à população. Portanto, o presente estudo teve por objetivo analisar a prevalência e as características epidemiológicas relacionadas ao trauma hepático nas vítimas encaminhadas ao Instituto Médico Legal (IML) de Palmas, Tocantins.

\section{MÉTODOS}

Um estudo transversal retrospectivo foi realizado no IML de Palmas, capital do estado de Tocantins, incluindo todas as vítimas encaminhadas para essa instituição, no período de janeiro de 2006 a dezembro de 2010, decorrentes de óbitos por causas violentas e que possuíssem lesões traumáticas de tecido hepático presentes, ainda que estas não fossem relatadas como sendo a causa mortis. A coleta de dados foi realizada pelos autores mediante análise dos registros do IML. Os dados avaliados foram sexo, idade, mecanismo do trauma (quando acidentes de trânsito, o tipo de veículo envolvido), tipos de trauma associado ao trauma abdominal, presença de lesões abdominais associadas ao trauma hepático, índice de gravidade de lesões conforme a escala AAST-OIS e número de óbitos cuja causa mortis foi o trauma hepático.

O estudo seguiu todos os preceitos éticos da Declaração de Helsinki e do Conselho Nacional de Saúde do Brasil, sendo aprovado pelo Comitê de Ética em Pesquisa com Seres Humanos da Universidade Federal do Tocantins, sob o parecer de número 020/2012. A coleta de dados consistiu em análise de arquivos, sem qualquer contato com as vítimas ou com seus familiares, sendo assim dispensado o termo de consentimento livre e esclarecido. Os autores seguiram as normas do IML de Tocantins, regidas judicialmente, não ocorrendo qualquer vazamento de informações sigilosas que envolvessem as vítimas.

Os dados foram inicialmente anotados em formulários elaborados pelos autores e posteriormente digitados em banco de dados e analisados por meio do programa Epi Info ${ }^{\circledR}$ 3.5.1 (Centers for Disease Control and Prevention, Atlanta, Estados Unidos), sendo calculadas as frequências absolutas e relativas.

\section{RESULTADOS}

Das 1.796 vítimas fatais avaliadas, obtiveram-se $241(13,4 \%)$ que apresentavam algum grau de trauma hepático. Destes, $206(85,5 \%)$ eram do sexo masculino. A principal faixa etária acometida foi dos 20 aos 40 anos, $134(55,6 \%)$. O tipo de trauma mais envolvido foi o contuso, com 179 casos $(74,3 \%)$, enquanto que o penetrante esteve presente em 59 (24,5\%). Três óbitos $(1,2 \%)$ não tiveram o tipo de trauma especificado.

Considerando todas as causas, a maioria dos óbitos, 135 (55,8\%), aconteceu durante os finais de semana (sexta-feira, sábado e domingo), nesses casos sendo mais envolvidos os jovens entre 21-30 anos, 47 casos $(58,1 \%)$. Observou-se que a principal causa de trauma hepático foram os acidentes de trânsito, tanto no sexo feminino, com 26 (74,3\%) casos, quanto no masculino, $117(56,8 \%)$. Esta causa foi a mais frequente em todas as faixas etárias. Considerando apenas os acidentes de trânsito, a maioria ocorreu também aos finais de semana, com $88(61,6 \%)$ casos, sendo que as motos estavam envolvidas em $53(37,0 \%)$ destes, e os carros em $42(29,4 \%)$. 
Em relação ao tipo de trauma, a principal causa de óbitos envolvendo trauma fechado foram ainda os acidentes de trânsito, 143 (79,9\%), enquanto entre os traumas penetrantes o ferimento por arma de fogo foi dominante, com $33(60 \%)$ casos (Tabela 1).

Tabela 1. Causas do trauma, de acordo com o mecanismo, envolvendo 241 vítimas fatais encaminhadas ao Instituto Médico Legal de Palmas, que evidenciaram trauma hepático. Palmas, Tocantins, Brasil, 2006-2010.

\begin{tabular}{lrc}
\multicolumn{1}{c}{ Variáveis } & N & (\%) \\
\hline Causas de trauma fechado & & \\
Total & 179 & $(100)$ \\
Acidente de trânsito (motocicleta ou automóvel) & 143 & $(79,9)$ \\
Atropelamento & 23 & $(12,9)$ \\
Quedas & 5 & $(2,8)$ \\
Agressão & 4 & $(2,2)$ \\
Outras causas & 4 & $(2,2)$ \\
Causas de trauma penetrante & & \\
Total & 59 & $(100)$ \\
Arma de fogo & 33 & $(60)$ \\
Arma branca & 26 & $(40)$ \\
Sem informação & 3 & \\
\hline
\end{tabular}

Em 147 (61\%) dos casos, as vítimas foram classificadas como politraumatizadas. Ao analisar o lobo hepático acometido, constatou-se que em 98 lesões $(40,7 \%)$, o lobo hepático direito fora lesionado, sendo que em $63(26,1 \%)$, mais de um lobo estava envolvido (Tabela 2).

Tabela 2. Distribuição de traumas associados ao trauma abdominal em casos de óbitos que apresentavam lesão hepática, e avaliação dos lobos hepáticos acometidos. Palmas, Tocantins, Brasil, 2006-2010.

\begin{tabular}{lrc}
\multicolumn{1}{c}{ Variáveis } & N & (\%) \\
\hline Traumas associados & 147 & $(61,0)$ \\
Politraumatizado* & 55 & $(22,8)$ \\
Trauma torácico & 14 & $(5,8)$ \\
Trauma cranioencefálico & 12 & $(5,0)$ \\
Trauma musculoesquelético & 10 & $(4,1)$ \\
Sem traumas associados & 2 & $(0,8)$ \\
Trauma raquimedular & 1 & $(0,4)$ \\
Trauma urogenital & & \\
Lobo hepático lesionado & 98 & $(40,7)$ \\
Lobo hepático direito & 63 & $(26,1)$ \\
Mais de um lobo acometido & 19 & $(7,9)$ \\
Lobo esquerdo & 2 & $(0,8)$ \\
Lobo caldado & 59 & $(24,5)$ \\
\hline Não especificado
\end{tabular}

* Dois ou mais sistemas envolvidos desconsiderando o trauma abdominal.
Entre os órgãos abdominais acometidos, a lesão esplênica foi a mais associada ao trauma hepático (Figura 1). Em relação à gravidade da lesão hepática, os graus II e III foram observados com maior frequência nos traumas contusos e penetrantes (Figura 2). Vinte e um óbitos $(8,7 \%)$ tiveram como causa direta o trauma hepático.

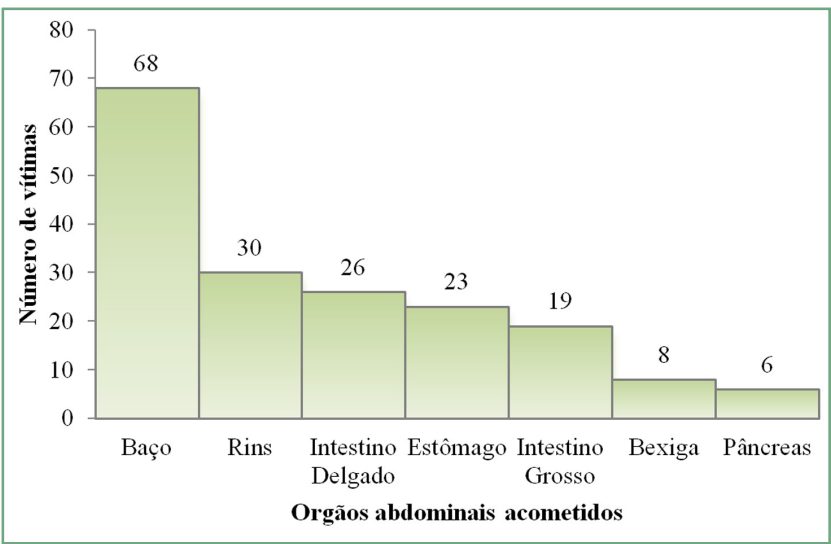

Figura 1. Número de vítimas que tiveram, além da lesão hepática, acometimento de outros órgãos abdominais. Palmas, Tocantins, Brasil, 2006-2010.

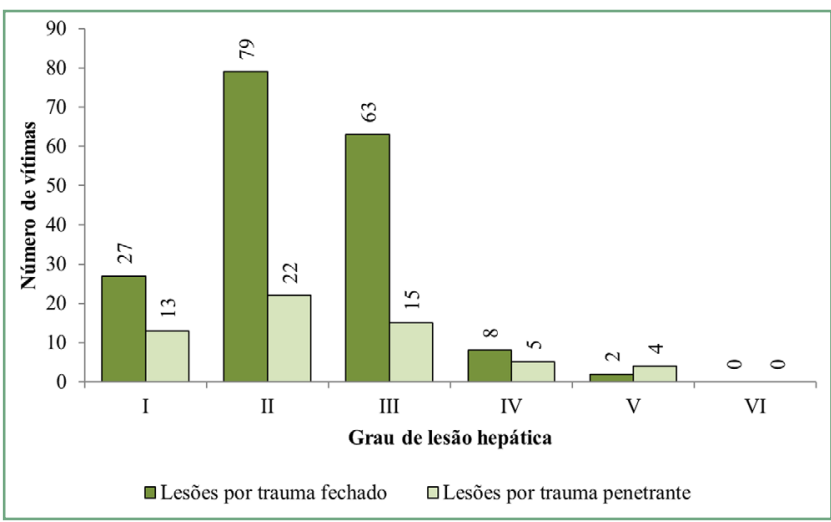

Figura 2. Número de vítimas fatais que apresentaram lesões hepáticas distribuídas conforme grau de lesão, determinada pela American Association for the Surgery of Trauma-Organ Injury Scale (AAST-OIS), e o mecanismo do trauma. Palmas, Tocantins, Brasil, 2006-2010.

\section{DISCUSSÃO}

O abdome é frequentemente acometido nos traumatismos, sendo que o fígado, devido ao seu tamanho e sítio anatômico, é um dos órgãos intraabdominais mais afetados, tanto por ferimentos penetrantes como por lesões contusas [3,8-10]. 
A distribuição entre trauma contuso ou penetrante varia em proporção de acordo com a região. Estudos realizados na Europa geralmente mostram índices de trauma contuso superiores aos índices de trauma penetrante, enquanto que estudos em países em desenvolvimento observam o contrário $[2,3]$. Um levantamento populacional de todos os casos de trauma hepático identificados na Escócia entre 1992 e 2002, em que foram avaliados 783 casos, mostrou que o trauma contuso ocorreu em $69 \%$, sendo a maioria deles por acidentes rodoviários [11]. Outros estudos europeus reafirmam a predominância do trauma contuso e, encontram índices maiores que $90 \%$ tanto em Estocolmo, na Suécia, quanto no Reino Unido [12-14]. Já no Brasil, um estudo em Curitiba mostrou que 54,6\% dos traumas foram penetrantes e, na África do Sul, encontraram um número ainda maior, $66 \%[15,16]$.

No presente estudo, o tipo de trauma mais frequente foi o contuso, aproximando-se mais da situação encontrada nos países desenvolvidos. Este resultado pode estar relacionado aos baixos índices de violência da cidade de Palmas, dado demonstrado no estudo de Walselfisz [17], em que esta cidade aparece em penúltimo lugar entre as capitais em relação às taxas de óbitos por arma de fogo, com um índice de 9,6 óbitos em 100.000 habitantes [17,18]. Além disso, ocorreu o aumento relativo de traumas contusos, podendo estar associado ao aumento do número de automóveis e motocicletas circulantes no país, que por sua vez, fazem um número cada vez maior de vítimas no trânsito [18].

No trauma fechado, a lesão hepática decorre de impacto direto, compressão entre o rebordo costal direito e a coluna vertebral ou devido às forças de aceleração e desaceleração súbita, como ocorre nos acidentes de trânsito. Por outro lado, quedas da própria altura podem ser importante causa de trauma hepático em idosos, possuindo outros mecanismos associados [3,4].

Pachter et al. [19], em estudo com 404 pacientes, observaram que $72 \%$ das lesões hepáticas foram ocasionadas por acidentes entre automóveis. O colégio Americano de Cirurgiões, através de seus bancos de dados, concluiu que, entre 1994 e 2003, 72,7\% dos traumas contusos foram causados por acidentes de trânsito [20]. Em concordância com estes dados, o presente estudo evidenciou que, entre os traumas fechados, cerca de $80 \%$ das lesões hepáticas foram ocasionadas por esse mesmo mecanismo. Corroborando com informações encontradas no cenário nacional, Palmas está em quarto lugar entre as capitais com maior número de óbitos em acidentes de trânsito, com uma taxa de 48,4 por 100.000 habitantes [21].
Zago et al. [22], em um estudo com 265 pacientes, evidenciaram que cerca de $35 \%$ dos traumas hepáticos estavam relacionados a acidentes com automóveis, enquanto as motocicletas estavam envolvidas em cerca de $25 \%$ [22]. Os mesmos autores, em outro estudo, com 748 pacientes, demonstraram que dentro dos traumas hepáticos fechados, 44,6\% foram relacionados com carros e $15 \%$ com motocicletas [2].

Segundo o Departamento Nacional de Trânsito (DENATRAN), em 2010 Palmas possuía, entre motocicletas e motonetas, um total de $37,9 \%$ do total da frota, enquanto que os automóveis representavam um total de $41,7 \%$ [23], justificando o maior envolvimento de motocicletas no trauma abdominal fechado envolvendo o fígado.

Entre as causas de trauma penetrante, o principal mecanismo foi o ferimento por arma de fogo, presente em $60 \%$ dos casos. Proporção semelhante foi encontrada no estudo de Zago et al. [2], no qual 67\% dos casos foram causados por esse mesmo mecanismo.

No contexto do politrauma, o trauma abdominal é um dos mais prevalentes. Além disso, outros estudos também mostram que lesões associadas são muito comuns no trauma hepático, e evidenciam números que variam de $75 \%$ até $90 \%$ [1,3,8,9]. Identificou-se nesse trabalho que a maior parte dos traumas hepáticos (61\%) apresentava-se em vítimas politraumatizadas. Esse cenário pode ser explicado pelo fato do principal mecanismo de trauma ser o acidente de trânsito.

Quanto às lesões associadas, os órgãos mais acometidos no presente estudo foram o baço em primeiro lugar, seguido de rins e intestino delgado, corroborando com diversas outras publicações que apresentam esses órgãos, juntamente com o fígado, como os principais atingidos no trauma abdominal $[8,24,25]$.

Segundo classificação AAST-OIS, as lesões mais leves correspondem àquelas até grau III; em média, $85 \%$ dos traumas hepáticos se enquadram dentro desta realidade $[3,26,27]$. O estudo em questão evidenciou que aproximadamente $90 \%$ das lesões, tanto contusas quanto penetrantes, apresentavam até grau III.

Dos lobos hepáticos, o direito é o mais comumente afetado por ser a porção do parênquima hepático mais volumoso. Já lesões do lobo esquerdo são raras e, quando presentes, geralmente estão associadas com impacto direto no abdome superior. As lesões do lobo caudado são extremamente raras e geralmente estão associadas a traumas de outros órgãos [4,15]. No presente estudo observou-se a predominância do lobo hepático direito, porém a coleta de dados foi prejudicada nesse quesito, pela escassez de informações na maioria dos documentos analisados. 
Houve um expressivo envolvimento do sexo masculino nos óbitos analisados com presença de trauma hepático; tal achado condiz com a realidade encontrada em outras fontes da literatura [2,3,9]. Em relação à idade, houve predominância de trauma hepático na terceira década de vida em ambos os sexos, o que novamente se assemelha a outros estudos, justificado pelo fato de nessa faixa etária haver maior prática de esportes, consumo de bebidas alcoólicas e envolvimento com agressões físicas. Alguns estudos apontam a quarta década como a de maior acometimento, sendo que todos concordam com o fato de que a população jovem em idade laboral é a mais acometida $[3,9,20]$.

$\mathrm{O}$ número de óbitos que ocorreram diretamente devido ao trauma hepático correspondeu a $8,7 \%$, semelhante ao observado em outros estudos, considerando formas de tratamento conservadoras e cirúrgicas, sem distinção entre as condutas [1, 28, 29].

As características epidemiológicas delineadas em Palmas descreveram o seguinte cenário: acometimento mais frequente de homens jovens na faixa etária laboral, com trauma hepático do tipo contuso, decorrente principalmente por acidente de trânsito, possuindo as motos como o mais importante fator envolvido. Entre os traumas penetrantes destacaram-se os ferimentos por arma de fogo. Segundo a AAIT-OIS, a maioria das lesões foram classificadas como leves, ou seja, até o grau III. Em sua maioria, as vítimas foram classificadas como politraumatizadas, possuindo como principal órgão envolvido o baço. Além disso, a maioria dos óbitos ocorreu aos finais de semana, período que sugere um maior consumo de bebidas alcoólicas, que poderia estar envolvido com os acidentes de trânsito. A porcentagem de óbitos cuja causa foi relacionada diretamente ao trauma hepático correspondeu a $8,7 \%$ de todos os casos. Os resultados encontrados podem contribuir para o planejamento de ações voltadas para a educação no trânsito, demonstrando às autoridades a necessidade de se investir nesse setor, visando a diminuir o número de óbitos de indivíduos ainda jovens.

\section{REFERÊNCIAS}

1. Lima SO, Cabral FLD, Neto AFP, Mesquita FNB, Feitosa MFG, Santana VR. Avaliação epidemiológica das vítimas de trauma abdominal submetidas ao tratamento cirúrgico. Rev Col Bras Cir. 2012;39(4):302-6. http://dx.doi.org/10.1590/S0100-69912012000400010

2. Zago TM, Pereira BM, Nascimento B, Alves MSC, Calderan TRA, Fraga GP. Trauma hepático: uma experiência de 21 anos. Rev Col Bras Cir. 2013;40(4):318-22. http://dx.doi.org/10.1590/S0100-69912013000400011

3. Stalhschmidt CMM, Formighieri B, Marcon DM, Takejima AL, Soares LGS. Trauma hepático: epidemiologia de cinco anos em um serviço de emergência. Rev Col Bras Cir 2008;35(4):225-8. http://dx.doi.org/10.1590/S0100-69912008000400004

4. Silvio-Estaba L, Madrozo-Gonzalez Z, Ramos-Rubio E. Actualización del tratamiento de los traumatismos hepáticos. Cir Esp. 2008;83(5):227-34. http://dx.doi.org/10.1016/S0009-739X(08)70559-1

5. Champion HR, Sacco WJ, Copes WS, Gann DS, Gennarelli TA, Flanagan ME. A revision of the Trauma Score. J Trauma.1989;29(5):623-9. http://dx.doi.org/10.1097/00005373-198905000-00017

6. Moore EE, Cogbill TH, Jurkovich GJ, Shackford SR, Malangoni MA, Champion HR. Organ injury scaling: spleen and liver (1994 revision). J Trauma. 1995;38(3):323-4. http://dx.doi.org/10.1097/00005373-199503000-00001

7. Benckert C, Thelen A, Gaebelein G, Hepp P, Josten C, Bartels M, Jonas S. Balanced management of hepatic trauma is associated with low liver-related mortality. Langenbeck's Arch Surg. 2010;395(4):381-6. http://dx.doi.org/10.1007/s00423-009-0566-9

8. Leite S, Taveira-Gomes A, Sousa H. Lesão Visceral em Trauma Abdominal: um estudo retrospectivo. Acta Med Port. 2013;26(6):725-30.

9. Ribas-Filho JM, Malafaia O, Fouani MM, Justen MS, Pedri LE, Silva LMA, Mendes JF. Trauma abdominal: estudo das lesões mais frequentes do sistema digestório e suas causas. ABCD Arq Bras Cir Dig. 2008;21(4):170-4. http://dx.doi.org/10.1590/S0102-67202008000400004

10. Diório AC, Fraga GP, Dutra Junior I, Joaquim JLGC, Mantovani M. Fatores preditivos de morbidade e mortalidade no trauma hepático. Rev Col Bras Cir. 2008;35(6):397-405. http://dx.doi.org/10.1590/S0100-69912008000600010

11. Scollay JM, Beard D, Smith R, McKeown D, Garden OJ, Parks R. Eleven years of liver trauma: the Scottish experience. World J Surg. 2005;29(6):744-9. http://dx.doi.org/10.1007/s00268-005-7752-x

12. Matsch T, Begquist D, Hedelin M, Findblack B. Leberverletzungen nachstumpfem Bauchtrauma. Unfallchircurgie. 1982;85:524-8.

13. Talving P, Beckman M, Haggmark T, Iselius L. Epidemiology of liver injuries. Scand J Surg. 2003;92(3):192-4.

14. Watson CJ, Calne RY, Padhani AR, Dixon AK. Surgical restraint in the management of liver trauma. Br J Surg. 1991;78(9):1071-5. http:// dx.doi.org/10.1002/bjs.1800780913

15. Von Bahten LC, Nicoluzzi JE, Olandoski M, Pantanali CA, Silva RFK. Trauma abdominal fechado: análise dos pacientes vítimas de trauma hepático em um Hospital Universitário de Curitiba. Col Bras Cir. 2005;32(6):316-20. http://dx.doi.org/10.1590/s0100-69912005000600006

16. Krige Je, Bornman PC, Terblanche J. Liver trauma in 446 patients. S Afr J Surg. 1997;35(1):10-5. 
17. Waiselfisz, JJ. Mapa da violência 2013. Mortes Matadas por armas de fogo. Centro Brasileiro de Estudos Latino-Americanos - CEBELA [Internet]. [Cited 2015 January]. Available from: http:/www.mapadaviolencia.org.br/pdf2013/MapaViolencia2013 armas.pdf

18. Carrasco CE, Godinho M, Berti ABM, Rizoli S, Fraga GP. Fatal motorcycle crashes: a serious public health problem in Brazil. World J Emerg Surg. 2012;7(1):S5. http://dx.doi.org/10.1186/1749-7922-7-S1-S5

19. Pachter HL, Knudson MM, Esrig B, Ross S, Hoyt D, Cogbill T, Sherman H, Scalea T, Harrison P, Shackoford S, Ochsner MG, Mucha, P. Status of nonoperative management of blunt hepatic trauma in 1995: a multicenter experience with 404 patients. J Trauma. 1996;40(1): 31-8. http://dx.doi.org/10.1097/00005373-199601000-00007

20. Hurtuk M, Reed RL 2nd, Esposito TJ, Davis KA, Luchette FA. Trauma surgeons practice what they preach: The NTDB story on solid organ injury management. J Trauma. 2006;61(2):243-54. http://dx.doi.org/10.1097/01.ta.0000231353.06095.8d

21. Waiselfisz, JJ. Mapa da violência 2013. Acidentes de trânsito e motocicletas. Centro Brasileiro de Estudos Latino-Americanos - CEBELA [Internet]. [Cited 2015 January]. Available from: http://www.mapadaviolencia.org.br/pdf2013/mapa2013_transito.pdf

22. Zago TM, Pereira BM, Calderan TRA, Hirano ES, Rizou S, Fraga GP. Trauma hepático contuso: comparação entre tratamento cirúrgico e não operatório. Rev Col Bras Cir. 2012;39(4):307-13. http://dx.doi.org/10.1590/S0100-69912012000400011

23. Ministério das Cidades, Departamento Nacional de Trânsito - DENATRAN - 2010.

24. Diercks DB, Clarke S. Initial evaluation and management of blunt abdominal trauma in adults. UpToDate. [Internet]. [Updated 2014 July; cited 2015 January]. Available from: http://www.uptodate.com

25. Saltzherr TP, van der Vlies CH, van Lienden KP, Beenen LF, Ponsen KJ,van Gulik TM. Improved outcomes in the non-operative management of liver injuries. HPB (Oxford). 2011;13:350-5. http://dx.doi.org/10.1111/j.1477-2574.2011.00293.x

26. Croce MA, Fabian TC, Menke PG, Waddle-Smith L, Minard G, Kudsk KA, Patton JH Jr, Schurr MJ, Pritchard FE. Nonoperative management of blunt hepatic trauma is the treatment of choice for hemodynamically stable patients. Results of a prospective trial. Ann Surg. 1995;221(6):744-53. http://dx.doi.org/10.1097/00000658-199506000-00013

27. Carrillo EH, Platz A, Miller FB, Richardson JD, Polk HC Jr. Non-operative management of blunt hepatic trauma. Br J Surg.1998;85(9): 461-8. http://dx.doi.org/10.1046/j.1365-2168.1998.00721.x

28. Fonseca-Neto OCL, Ehrhardt R, Miranda AL. Estudo da morbimortalidade em pacientes com trauma hepático. ABCD Arq Bras Cir Dig. 2013;26(2):129-32. http://dx.doi.org/10.1590/S0102-67202013000200012

29. Neves GG, Schneider LP, Malutta SS, Pimentel SK, Neto AB. Trauma hepático: principais características e importância do tratamento conservador. Panam J Trauma Crit Care Emerg. 2012;1(3):188-90. http://dx.doi.org/10.5005/jp-journals-10030-1041 